\title{
From Cascade to Spike—a Fractal-Geometry Approach
}

\author{
Y.-T. Cheng, ${ }^{(a)}$ M.-A. Nicolet, and W. L. Johnson \\ California Institute of Technology, Pasadena, California 91125
}

(Received 10 November 1986)

\begin{abstract}
The fractal nature of collisional cascades in solids is investigated by means of a model of "idealized" collisional cascade and the Winterbon-Sigmund-Sanders theory of atomic collisions. It is shown that the fractal dimensionality increases as the cascade evolves because of the change of the effective interaction potential. The condition of a spike is shown to arise naturally from the concept of "space-filling" fractals. The characteristic kinetic energy per particle in a spike is estimated to be on the order of a few electronvolts.
\end{abstract}

PACS numbers: $61.80 . \mathrm{Jh}, 05.40 .+\mathrm{j}, 34.10 .+\mathrm{x}$

Cascades and spikes are two concepts frequently used in describing ion-solid interactions. ${ }^{1,2}$ As an energetic ion penetrates a solid, it transfers part of its energy by colliding with target atoms. These atoms recoil and collide with other atoms, resulting in higher generations of collisions. The later-generation collisions produce many low-energy recoils which induce small displacements in random directions. The successive generations of the collisional process form a collisional cascade. ${ }^{1}$ A spike or a high-density cascade has been frequently described as a limited volume with the majority of atoms temporarily in motion as a result of collisions. ${ }^{2}$ A thermal spike is a spike in which the velocity distribution of moving particles resembles the Maxwell-Boltzmann distribution. The concept of temperature can then be introduced locally by the law of equipartition.

Cascade and thermal-spike models have been applied to radiation-damage problems, ${ }^{1,2}$ such as creation of defects, sputtering, and, more recently, ion implantation and ion-beam mixing. ${ }^{3}$ However, questions still remain as to what a spike is and, in particular, how a cascade evolves into a spike. In this Letter, we shall show that the notion of fractal dimensionality, introduced by Mandelbrot, ${ }^{4}$ provides a natural framework for the study of these problems. The idea of fractal dimension has had an impact on a series of problems ranging from coastline geometry, to disordered materials, to galaxy formation. ${ }^{4,5}$ It has also been recognized that the idea of fractals might be of interest to atomic collisions in solids. ${ }^{6}$

In Fig. 1, we plot "trees" that are similar to those in Ref. 4. The trees have infinitely thin stems, and the same angle $\theta=\theta_{1}+\theta_{2}$ between the branches at every branching point. For each tree, the length ratio between the successive branches, $\gamma$, is fixed throughout, and the fractal dimension is $D=\ln 2 / \ln (1 / \gamma){ }^{4}$ In Figs. 1(a) and (b), $D$ has values 1 and 2, respectively. Trees with larger values of $D$ are more "space-filling" than trees with smaller $D .{ }^{4}$

A natural connection between a tree and a collisional cascade is established if each branching point of a tree is taken to represent a binary collision event, and each branch corresponds to a trajectory between collisions.

In real collisional cascades, as well as computergenerated ones, ${ }^{7}$ the scattering angle and the distance traveled between collisions may be considered stochastic.

(a)

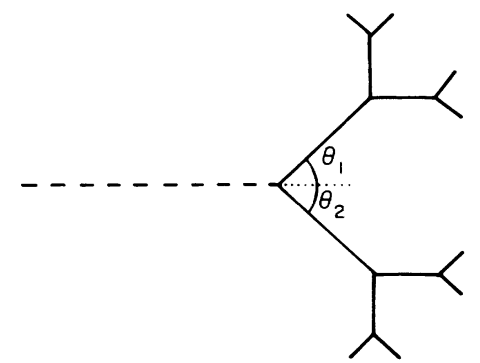

(b)

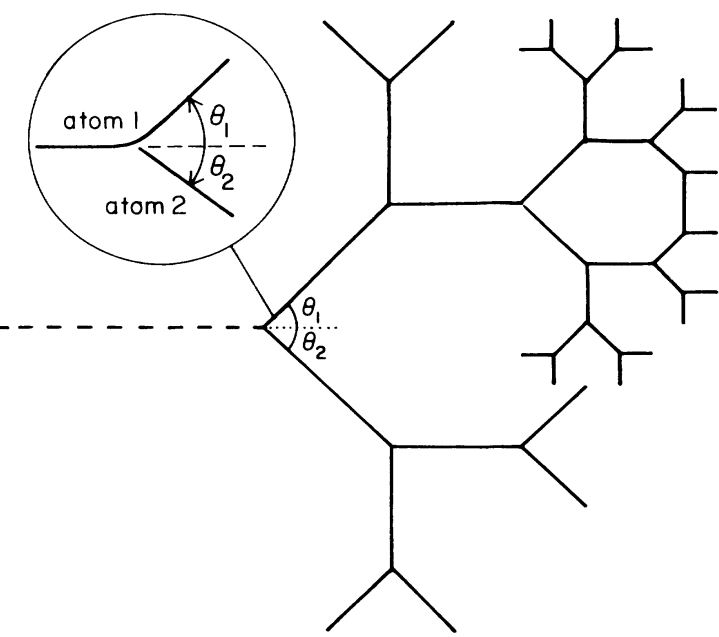

FIG. 1. Fractal trees (solid branches) with (a) $\gamma=\frac{1}{2}$, so that $D=1$, and (b) $\gamma=\left(\frac{1}{2}\right)^{1 / 2}$, so that $D=2$. They also represent the idealized collisional cascades (a) for $V(r) \propto r^{-2}$, and (b) for $V(r) \propto r^{-4}$. The cascades (trees) must be terminated in the two cases when the mean free path (branch length) approaches the same predetermined shortest length scale. In the $D=2$ case, (b), only one section of the "planefilling" cascade (tree) is shown. 
Consequently, useful information can only be obtained from statistical averages. It is, therefore, instructive to construct a cascade in which the scattering angle and the distance traveled between collisions at each generation take their average values. This idealization of cascades is in the same spirit as Mandelbrot's treatment of the coastline problem in which an irregular coastline is first approximated by a Koch curve. ${ }^{4}$

To construct an idealized collisional cascade, we begin with the hard-sphere collision approximation in conjunction with inverse-power potential $V(r) \propto r^{-1 / m}$, where $r$ is the internuclear distance and $0 \leq m \leq 1 .{ }^{8,9}$ In the study of collisions with large scattering angle and large momentum and energy transfer, this procedure has often been used as a convenient starting point. ${ }^{9,10}$ Later we shall show that the result of this analysis is general and is not limited to the hard-sphere collision approximation. Consider a system made of identical particles. At each energy concerned, the radius of the particles is defined as half of the distance of closest possible approach $\rho_{0}$ when the impact parameter for binary collisions is 0 and the scattering angle $\phi$ in the center-of-mass (c.m.) coordinate system is $\pi$. For that case, the potential energy $V$ at $\rho_{0}$ is given by ${ }^{9}$

$$
V\left(\rho_{0}\right)=\frac{1}{2} E_{0}
$$

where $E_{0}$ is the incident particle kinetic energy in the laboratory coordinate system (LC). Equation (1) defines $\rho_{0}$. Following the derivation in Ref. 9, the differential and total cross sections are given by

$$
d \sigma=\pi \rho_{0}^{2} d E_{1} / E_{0}
$$

and

$$
\sigma=\pi \rho_{0}^{2}
$$

where $E_{1}$ is the kinetic energy of the recoil particle in laboratory coordinates. The mean recoil energy $\bar{E}_{1}$ is given by $\bar{E}_{1}=\frac{1}{2} \bar{E}_{0}$. We can generalize this result to the $n$th generation of collisions, i.e.,

$$
\bar{E}_{n}=\frac{1}{2} \bar{E}_{n-1}
$$

From Eq. (3), we obtain the average scattering angle in the c.m. system, $\bar{\phi}=\frac{1}{2} \pi$. In the LC system, the average scattering angles become $\theta_{1}=\theta_{2}=\frac{1}{4} \pi$.

The mean free path of the $n$th generation is given by $\lambda_{n}=1 / N \sigma_{n}$, where $N$ is the atomic density. For inversepower potentials, $V(r)=G r^{-1 / m}$, with $G$ a constant which depends on $m$, and $0 \leq m<1$, the total effective cross section ${ }^{11} \sigma_{n}$ equals $\pi\left(2 G / \bar{E}_{n}\right)^{2 m}$, according to Eqs. (1) and (2b). We obtain

$$
\begin{aligned}
\lambda_{n} & =(1 / N \pi)\left(\bar{E}_{n} / 2 G\right)^{2 m} \\
& =\left(\frac{1}{2}\right)^{2 m}(1 / N \pi)\left(\bar{E}_{n-1} / 2 G\right)^{2 m} \\
& =\left(\frac{1}{2}\right)^{2 m} \lambda_{n-1},
\end{aligned}
$$

where we have used Eq. (3). The mean free path ratio, $\gamma=\lambda_{n} / \lambda_{n-1}=2^{-2 m}$, is independent of $n$, the generation of the cascade. The collisional cascade generated by $\gamma=2^{-2 m}$ and the mean scattering angle $\theta=\frac{1}{4} \pi$ is therefore self-similar and has a fractal dimension

$$
D=\frac{\ln 2}{\ln (1 / \gamma)}=\frac{1}{2 m}
$$

Thus, within the approximation used here, the fractal dimensionality of a cascade depends explicitly on the type of interatomic potential encountered. For the inverse-square potential $m=\frac{1}{2}$ we have a cascade of fractal dimension 1. For the inverse-power potential with $m=\frac{1}{6}$, we have a cascade of fractal dimension 3, which equals the physical dimension of the space in which an actual collisional cascade is embedded. For $\frac{1}{2}<m \leq 1,1>D \geq \frac{1}{2}$ from Eq. (5). This is the case of "subfractal trees" 4 and $D$ is then necessarily 1 which is the topological dimension of a branching tree (see Ref. 4, pp. 152-153). In particular, $D=1$ for Rutherford scattering $(m=1)$. As examples, idealized collisional cascades for $V(r) \propto r^{-2}$ and $V(r) \propto r^{-4}$ are those trees shown in Fig. 1.

A "spike" is, as has been frequently described, a limited volume with the majority of atoms temporarily in motion. ${ }^{2}$ This spike condition is ambiguous because the terms "limited volume" and "majority" cannot be uniquely defined for a treelike structure (see Fig. 1). Thus, the density of moving particles cannot be defined without further assumptions. Such difficulties as specifying the cascade volume have been recognized by Sigmund. ${ }^{12}$ To circumvent these difficulties, we propose an alternative approach to the spike condition by applying a well-defined concept of space-filling fractals ${ }^{4}$ : A fractal is space filling when its fractal dimension $D$ equals the physical dimension $E$ of the space in which it is embedded.

This space-filling condition is illustrated in the following for the case of idealized collisional cascades. We first identify the shortest length scale to be the shortest mean free path $\lambda_{g}$ and equate this to the interatomic spacing $a$, i.e., $\lambda_{g}=a$. Likewise, we take the longest length scale to be the longest mean free path $\lambda_{0}\left(>\lambda_{g}\right)$. For an idealized collisional cascade of dimension $D$ $=\ln 2 / \ln (1 / \gamma)$ the total number of generations of cascades required to reduce the mean free path from $\lambda_{0}$ to $\lambda_{g}$ is given by

$$
g=\frac{\ln \left(\lambda_{g} / \lambda_{0}\right)}{\ln \gamma}
$$

and the total number of displaced atoms $N_{c}$ is given by

$$
N_{c}=2^{g}=\left(\lambda_{0} / \lambda_{g}\right)^{D} \text {. }
$$

An upper bound $L$ for the linear dimension of the cascade can be obtained by summing up the series: $\sum_{i=0}^{g} \lambda_{i}$ $\leq \sum_{i=0}^{\infty} \lambda_{i}=\lambda_{0} /(1-\gamma)$; thus, $L=\lambda_{0} /(1-\gamma)$. Let us 
suppose that the cascade is embedded in a physical space of dimension $E$. The total number of lattice points $N_{a}$ included in the volume defined by an $E$-dimensional sphere of radius $L$ can be calculated. We find

$$
N_{a}=\left(L / \lambda_{g}\right)^{E}=\left(\lambda_{0} / \lambda_{g}\right)^{E}[1 /(1-\gamma)]^{E} .
$$

The ratio $\eta=N_{c} / N_{a}$ between the total number of displaced atoms and the total number of lattice atoms in the $E$-dimensional sphere of radius $L$ can be calculated from Eqs. (7) and (8). In the limit as $\lambda_{0} \rightarrow \infty$, this ratio becomes

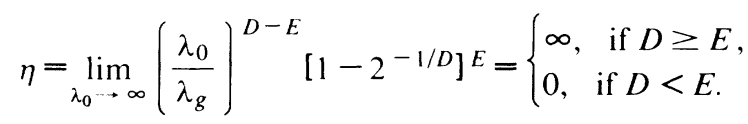

This confirms that $D=E$ is indeed a critical dimension for a space-filling collisional cascade.

Next, we point out that the notion of a dimensionality $D=1 / 2 m$ for collisional cascades governed by an inverse-power potential $V(r) \propto r^{-1 / m}$ is inherent in the Winterbon-Sigmund-Sanders (WSS) theory of collisional cascade. ${ }^{13}$ From the WSS theory (which is formulated in three-dimensional physical space), the characteristic length scale (e.g., ion range) $R$ of a collisional cascade scales with the incident ion energy $E_{0}$ and is given by ${ }^{13}$

$$
R=E_{0}^{2 m} / N C_{m}
$$

where $C_{m}$ is a constant for fixed $m$. With a KinchinPease type of argument, ${ }^{1,14}$ the number of displaced atoms $N_{c}$ is given by

$$
N_{c} \propto R^{1 / 2 m}
$$

From the equivalent definitions of fractal dimension, 4,5 we recognize that $\mathrm{Eq} .(11)$ is just another manifestation of the fractal nature of the underlying collisional cascade with the same fractal dimension $D=1 / 2 \mathrm{~m}$ obtained from the idealized cascade approach [Eq. (5)]. Similarly to the discussion leading to Eq. (9), we examine the ratio $\eta$ between the displaced atoms to the lattice atoms:

$$
\eta \propto \lim _{R \rightarrow \infty} R^{D-3}=\left\{\begin{array}{l}
\infty, \text { if } D \geq 3, \\
0, \text { if } D<3 .
\end{array}\right.
$$

Thus, $D=3$ is again shown to be the critical dimension of a space-filling collisional cascade. Conclusion 1: A collisional cascade governed by the inverse-power potential $V(r) \propto r^{-1 / m}(0 \leq m \leq 1)$ is a fractal with a fractal dimension $D=1 / 2 m$ for $0 \leq m \leq \frac{1}{2}$ and $D=1$ for $\frac{1}{2}<m \leq 1$. In three-dimensional space, a spacefilling collisional cascade occurs when $0<m \leq \frac{1}{6}$.

As an actual collisional cascade evolves in time, the characteristic kinetic energy of atoms or ions changes from the primary incident ion energy, which is typically on the order of several hundred kiloelectronvolts in most of the ion-mixing or ion-implantation experiments, to the electronvolt energy range. Inverse-power potentials $V(r) \propto r^{-1 / m}$, with $0 \leq m<1$, have been frequently utilized to model collisional dynamics. ${ }^{8}$ For example, it has been argued that over a major portion of the kiloelectronvolt range and for medium- to heavy-mass ions and atoms, the inverse-square potential (i.e., $m=\frac{1}{2}$ ) is a fair approximation, while in the lower kiloelectronvolt and upper electronvolt region, $m=\frac{1}{3}$ should be adequate. In the electronvolt region, $m$ may be taken close to zero. ${ }^{8}$ To describe an actual collisional cascade from beginning to end by the inverse-power potential, decreasing values of $0 \leq m<\frac{1}{2}$ must be used. The actual cascade is, therefore, not a fractal in the sense which has been given so far, but is a nonuniform fractal $^{4}$ with a variable dimensionality which increases from its initial dimensionality 1 (i.e., $m=\frac{1}{2}$ ). Conclusion 2: In an actual collisional cascade, the fractal dimensionality increases as the cascade evolves because of the change of the interaction potential.

From the analysis of space-filling collisional cascades, it is appropriate to postulate that, in three-dimensional space, a transition from cascade to spike occurs when the fractal dimension of a collisional cascade $D$ equals 3 . The condition $D=3$ implies $m=\frac{1}{6}$. This corresponds to a potential $V(r) \propto r^{-6}$, which is, according to the previous discussions, applicable to particles of a characteristic kinetic energy in the "upper electronvolt to electronvolt range. ${ }^{8}$ Conclusion 3: In three-dimensional space, a spike, or a "space-filling" cascade, occurs at a characteristic particle kinetic energy on the order of a few electroniolts per particle.

Recently, there have been reports that spikes indeed occur at an energy level of about a few electronvolts per particle. ${ }^{3,15}$ In ion-mixing experiments, ${ }^{3}$ it was shown that thermal-spike effects, such as the chemically biased diffusion due to the heat of mixing of a binary alloy, occur at a characteristic kinetic energy level of about an electronvolt per particle. In a computer simulation, ${ }^{15}$ it was observed that the equipartition of kinetic and potential energy of moving particles occurs in about the electronvolt-per-particle range, thus indicating the approach to thermal equilibrium within the cascade at this characteristic energy. These findings are consistent with the above conclusion 3. In addition, the numerical value of the present estimate for the energy density of spikes falls between the values obtained by Sigmund. ${ }^{12}$ The latter values are based on the estimates of the spike volume. This procedure, as Sigmund pointed out, "is not easily specified both in principle and in practice." 12 Conclusion 3 above, however, is reached without specific assumptions regarding the measure of the cascade volume.

We have examined the fractal nature of collisional cascades by studying the idealized collisional cascade and the WSS theory. Either approach enables us to es- 
tablish a fractal dimension for cascades governed by the inverse-power potential. The idealized collisionalcascade approach shows, at the level of binary-collision events, the scaling property of the mean free path [Eq. (4)] from which the fractal dimensionality results. The approach based on the WSS theory has the advantage of not relying on assumptions such as the hard-sphere collision approximation, though the underlying feature which gives rise to the fractal dimension is obscured. The fact that both approaches give the same result suggests that the two methods are complementary to each other and the conclusions drawn from either approach are of general nature. In summary, we have shown that in actual collisional cascades the fractal dimensionality of the branching process increases as the cascade evolves because of the change of the interaction potential with energy; a spike occurs when the fractal dimension of the cascade equals the physical dimension of the solid and this corresponds to an energy scale of a few electronvolts per particle.

The authors would like to thank Professor N. R. Corngold of California Institute of Technology, Professor T. Egami of the University of Pennsylvania, and Dr. M. Van Rossum of Interuniversitair Micro-Elektronica Centrum (Belgium) for helpful discussions. This work was supported by the U.S. National Science Foundation Grant No. DMR-84-21119.

\footnotetext{
(a) Present address: Physical Chemistry Department, General Motors Research Laboratories, Warren, MI 48090.

${ }^{1}$ G. H. Kinchin and R. S. Pease, Rep. Prog. Phys. 18, 1 (1955); P. Sigmund and A. Gras-Marti, Nucl. Instrum.
}

\section{Methods 182/183, 25 (1981)}

${ }^{2}$ F. Seitz and J. S. Koehler, Prog. Solid State Phys. 2, 305 (1956); P. Sigmund, Appl. Phys. Lett. 25, 169 (1974); D. A. Thompson, Rad. Effects 56, 105 (1981).

${ }^{3}$ Y.-T. Cheng, M. Van Rossum, M.-A. Nicolet, and W. L. Johnson, Appl. Phys. Lett. 45, 185 (1984); W. L. Johnson, Y.-T. Cheng, M. Van Rossum, and M.-A. Nicolet, Nucl. Instrum. Methods Phys. Res., Sect. B 7/8 657 (1985).

${ }^{4}$ B. B. Mandelbrot, The Fractal Geometry of Nature (W. H. Freeman, New York, 1983).

${ }^{5}$ Kinetics of Aggregation and Gelation, edited by F. Family and D. P. Landau (North-Holland, Amsterdam, 1984); S. H. Liu, in Solid State Physics, 39, 207 (1986); On Growth and Form, edited by H. E. Stanley and N. Ostrowsky (Martinus Nijhoff, Boston, 1986).

${ }^{6}$ See, e.g., K. B. Winterbon, Rad. Effects 60, 199 (1982).

${ }^{7}$ J. R. Beeler, Jr., Phys. Rev. 150, 470 (1966); F.-Z. Cui and H.-D. Li, Nucl. Instrum. Methods Phys. Res., Sect. B 7/8, 650 (1985).

${ }^{8}$ J. Lindhard, V. Nielsen, and M. Scharff, Kgl. Dan. Vidensk. Selsk. Mat.-Fys. Medd. 36, No. 10 (1968); P. Sigmund, Phys. Rev. 184, 383 (1969).

${ }^{9} \mathrm{M}$. W. Thompson, Defects and Radiation Damage in Metals (Cambridge Univ. Press, London, New York, 1969).

${ }^{10}$ N. Bohr, Mat. Fys. Medd. Dan. Vid. Selsk. 18, No. 8 (1948); L. D. Landau and E. M. Lifshitz, Mechanics (Pergamon, Oxford, 1976).

"The term "total effective cross section" is adopted from Bohr, Ref. 10.

${ }^{12} \mathrm{P}$. Sigmund, in Sputtering by Particle Bombardment I, edited by R. Behrisch (Springer-Verlag, Berlin, 1981).

${ }^{13}$ K. B. Winterbon, P. Sigmund, and J. B. Sanders, Mat. Fys. Medd. Dan. Vid. Selsk. 37, No. 14 (1970).

${ }^{14}$ P. Sigmund, Appl. Phys. Lett. 14, 114 (1969); P. Sigmund, Rad. Effects 1, 15 (1969).

${ }^{15}$ W. E. King and R. Benedek, J. Nucl. Mater. 117, 26 (1983). 